\title{
Secure Communication Using 4-D Arrays for Cognitive Radio
}

\author{
K. Vara Prasad ${ }^{1}$, P. Satyanarayana ${ }^{2}$, A. Kusuma mounica ${ }^{3}$ \\ Assistant Professor, Department of ECE, V R Siddhartha Engineering College, A.P, Vijayawada ${ }^{1,2}$ \\ Project Student, Department of ECE, V R Siddhartha Engineering College, A.P, Vijayawada ${ }^{3}$
}

\begin{abstract}
In recent days, the growing request of information and mobility has an unavoidable impact on efficient utilization of wireless systems and services with a consequent congestion of wireless medium. As a consequence, issues regarding an efficient and smart utilization of limited radio resource have been raised. To address these problems, a proposed method is the use of antenna systems that are able to first sense the external EM environment and then reconfigure the radiation characteristics of the generated field to guarantee a reliable communication by using time modulation in 4-D arrays. Harmonic radiations having different spatial distribution and shapes can be synthesized in order to receive signals arriving on the antenna from different directions. The capability to generate a field having different frequencies in spatial distribution implies that signal transmitted by time modulated 4-D arrays is direction dependent. Such a feature is exploited in this paper to implement a secure communication scheme directly at the physical layer.
\end{abstract}

Keywords: 4-D arrays, Cognitive radio, RF switches, Time Modulation, Harmonic Radiations.

\section{INTRODUCTION}

The primary objectives of CR are the efficient use of the available spectrum and the reliability of the wireless communications. In order to achieve these goals, the antenna has to rapidly adapt to the changing electromagnetic scenario by reconfiguring, possibly via software (SW), the radiation patter in order to suppress the interfering signals, to transmit/receive information in the free/less-distributed bands and to properly modulate the signals to obtain secure communication links.

In the last decades, several solutions have been proposed in the scientific literature to the design and synthesis of adaptive/smart antennas, $\mathrm{SW}$-defined radio system and directional modulation techniques [1]-[3] as suitable ways to implement CR. There has been recently a renewed interest towards the use of time as an additional degree of freedom (DoF) for the antenna synthesis to obtain performance not achievable with conventional antennas.

As a matter of fact, these power losses unavoidably reduce the antenna directivity since they have to be filtered out to correctly receive the desired signal. More recently, the research interest in time-modulated 4-D arrays has been redirected towards making use of these harmonic radiations. The synthesis of simultaneous and multiple harmonic beams for applications like direction finding and beam steering has been already investigated [8], [9] to improve the reliability of the communication system. Indeed, the individual harmonic patterns, each associated with a characteristic frequency, can be utilized as independent information channels which may be separated at the receiver and each one utilized in a conventional manner. This key feature of 4-D arrays enables their use for simultaneous operations and potentially to harvest multiple replicas of a signal thus leading to their applications in multiple-input multiple-output (MIMO) systems. The same antenna structure can also be used to receive multiple independent signals which carry different information. As a matter of fact, it is widely recognized that the use of MIMO antennas can be profitably adopted to increase both the spectral and spatial efficiency of wireless communications as well as the system throughput as requested in CR systems.

\section{SECURE COMMUNICATION}

One characteristic of cognitive radio is to provide secure and reliable communication using spread spectrum modulation and encryption techniques. However, it is still possible in theory for a receiver at an undesired direction to eavesdrop the information. To avoid this drawback, techniques based on the use of variable directional antenna (e.g., electrically steerable parasitic array radiatorESPAR) and channel estimation for secret key generation and near-field antenna modulation strategies have been presented [11]. An improved key generation method uses multi-level quantization and parity check in the secret key agreement scheme. It enhances key agreement probability in legitimate parties efficiently without reducing the security. There was a trade-off between the key agreement probability and the security. This method realizes higher security than the conventional one in the key agreement system. More recently, Directional Modulation techniques have been proposed to provide secure communication link [1]-[3], [10].

\section{PROPOSED METHOD}

In previous studies on signal-processing of 4-D arrays, the center frequency and sideband signals are usually separated from each other to avoid the aliasing effects (overlapping of the modulated signal spectrum) [4]. Thus, the signal bandwidth $\mathrm{B}$ is usually set less than the time modulation frequency $f_{p}$ such that the original signal can 
be recovered using a band-pass filter, whose bandwidth is a little greater than $\mathrm{B}$ and less than $\mathrm{f}_{\mathrm{p}}$,

In secure communications based on 4-D arrays, the aliasing effects are utilized for the purpose of distorting the radiated signals in some directions where sideband signals exist. Accordingly, suppose that a signal with a bandwidth $B\left(B>f_{p}\right)$ is to be transmitted by the 4-D array [Fig.1(b)]. If the radiated signal in a specific direction of the array is not time-modulated, the frequency spectrum of the signal is the same as that of original signal. However, the radiated signals in other side lobe directions have distorted frequency spectrum, as shown in Fig.4. Obviously, the signals cannot be recovered fully by using any bandwidth filter, and their time-domain waveforms will become distorted.

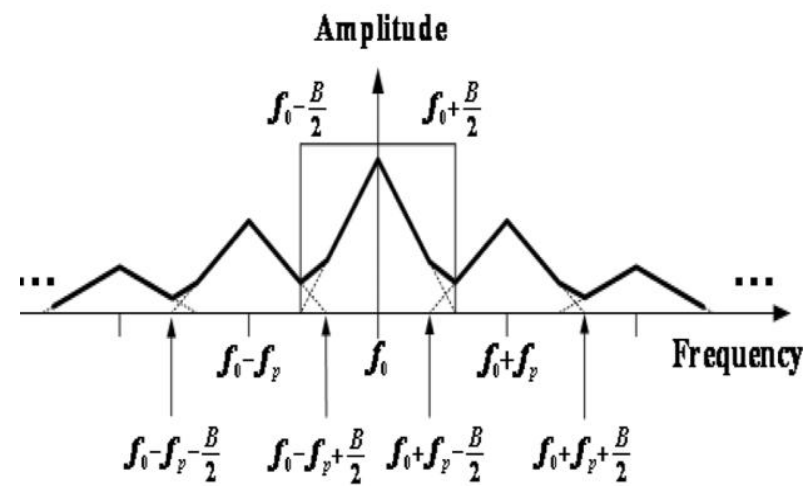

Fig.4. Frequency spectrum of the transmitted signals in 4$D$ arrays when $B>f_{p}$

As an example, a broadside 4-D array of $\mathrm{N}=8$ isotropic elements with $d=\lambda / 2$ at the operating frequency $f_{0}$ is considered. The array is excited with uniform amplitude and phase, operating as a transmitting antenna. The time modulation period of the array is supposed to be $T_{p}$, implying a time modulation frequency $\mathrm{f}_{\mathrm{p}}=1 / \mathrm{T}_{\mathrm{p}}$.

Time-modulated linear arrays (TMLAs) based on singlepole-single-throw (SPST) switches [12] usually have higher reduction in gain due to the sideband radiation and power absorption of the off state absorptive switches. The gain of TMLA can be improved significantly by controlling two array elements with each SPDT switch. Moreover, the TMLA has a constant instantaneous directivity, which is favourable for some applications. A 4-D array with constant directivity has been obtained by switching-on an element while switching-off another element. Therefore, a typical time modulation function $\mathrm{U}_{\mathrm{n}}(\mathrm{t})$ is designed as

$$
\begin{gathered}
\mathrm{U}_{\mathrm{n}}(\mathrm{t})\left\{\begin{array}{l}
\text { For } \mathrm{n}=1,8 \\
1,0.0 \leq \mathrm{t} \leq 0.4 \mathrm{~T}_{\mathrm{p}} \\
0, \text { others }
\end{array}\right. \\
\mathrm{U}_{\mathrm{n}}(\mathrm{t}) \text { f } \begin{array}{c}
\text { For } \mathrm{n}=2,7 \\
1,0.4 \mathrm{~T}_{\mathrm{p}}<\mathrm{t} \leq 1.0 \mathrm{~T}_{\mathrm{p}} \\
\text { others }
\end{array} \\
\mathrm{U}_{\mathrm{n}}(\mathrm{t})=\left\{\begin{array}{l}
\text { For } \mathrm{n}=3,4,5,6 \\
\left\{1,0.0 \mathrm{~T}_{\mathrm{p}}<\mathrm{t} \leq 1.0 \mathrm{~T}_{\mathrm{p}}\right.
\end{array}\right.
\end{gathered}
$$

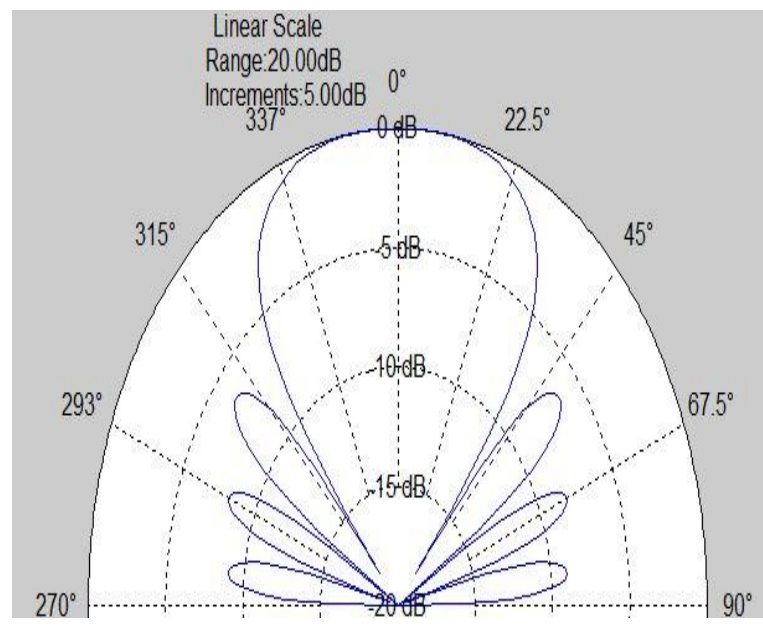

Fig.5. Radiation pattern of Uniform Linear Array

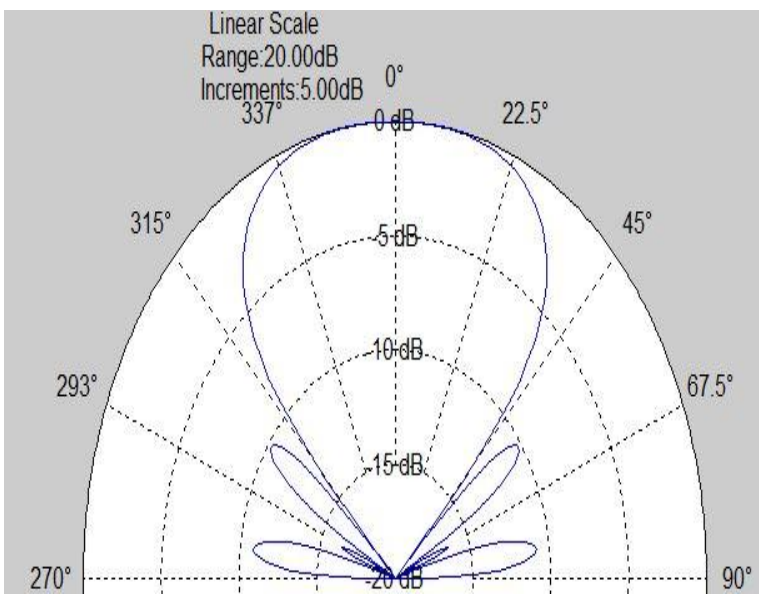

Fig.6. Radiation pattern generated at $\mathrm{h}=0$

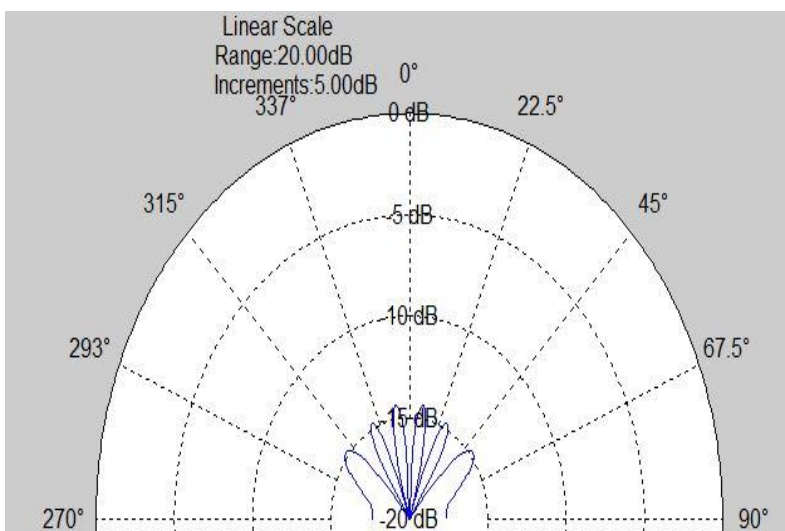

Fig.7. Radiation pattern generated at $\mathrm{h}=1$

The radiation pattern of a uniform linear array is shown in Fig.5. The radiation patterns of the 4-D array at the centre frequency as well as the first two harmonics are shown in Fig.6, Fig.7 \& Fig.8. It can be seen that the side lobe level decreases on account of time modulation compared to that in a normal uniform linear array. Also, it is seen that a null of sideband patterns appear at broadside $\left(\theta=0^{0}\right)$, implying that the signal at this angle is not modulated (no sideband signals). However, the transmitted signals in the other directions except for the nulls are time-modulated. According to Fig.6, Fig.7 \& Fig.8 the sideband signals exist in any direction except for the broadside and nulls. 


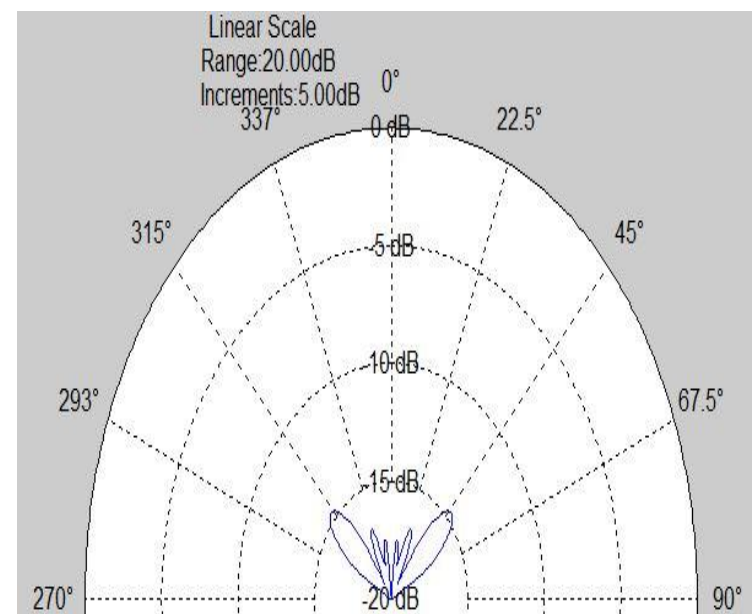

Fig.8. Radiation pattern generated at $\mathrm{h}=2$

It can be anticipated in theory that the transmitted signal in any direction except for the broadside will be distorted. Thus, this characteristic is suitable for secure communication.

To provide secure communication using time-modulated 4-D arrays, AM signal transmission through the 4-D array is presented. The expression of a double-sideband AM signal can be modeled as

$$
\mathrm{S}_{\mathrm{AM}}(\mathrm{t})=\left[1+\cos \left(2 \pi \mathrm{f}_{\mathrm{s}} \mathrm{t}\right)\right] \mathrm{e}^{\mathrm{j} 2 \pi \mathrm{f} 0 \mathrm{t}}
$$

where $f_{s}$ represents the amplitude modulation frequency.

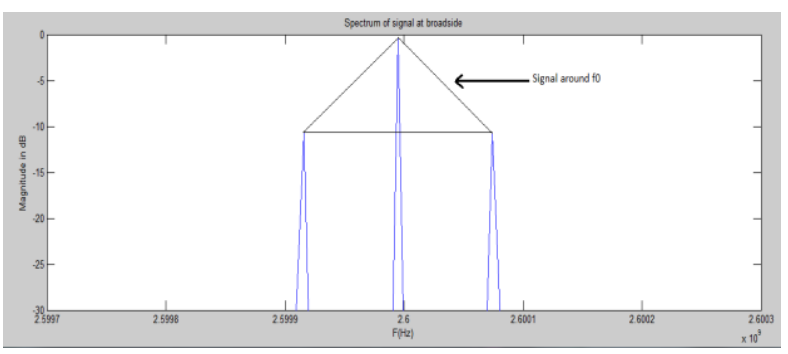

Fig.10. Frequency spectrum of the transmitted signal from the 4-D array at $0^{0}$

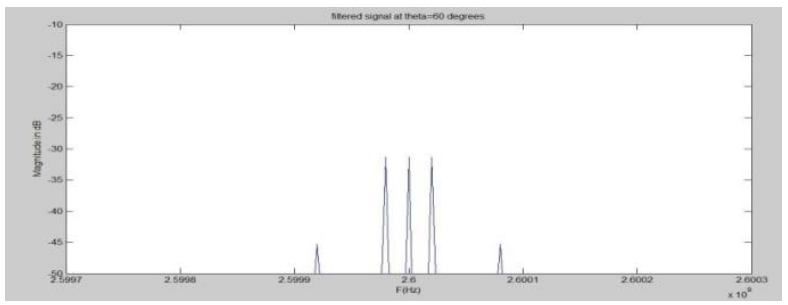

Fig.12. Frequency spectrum of the transmitted signal from the $4-\mathrm{D}$ array at $60^{\circ}$ after filtering

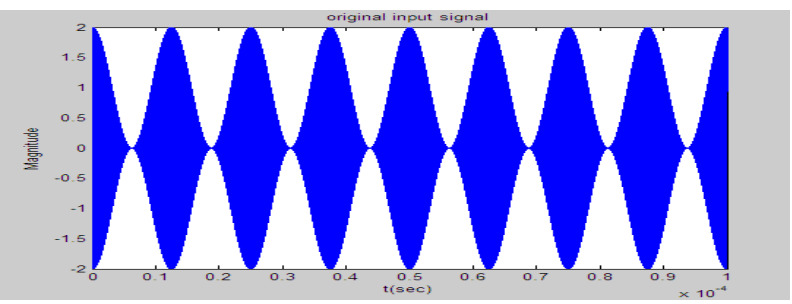

Fig.13. Simulated AM signal waveform: original AM signal

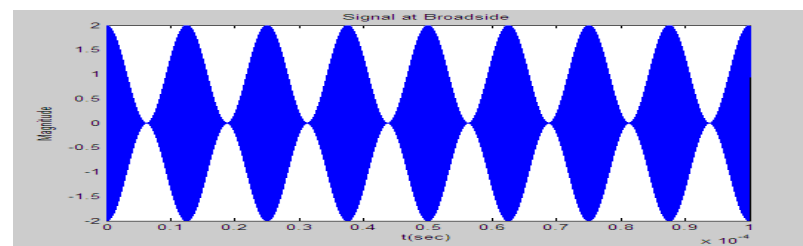

Fig.14. Simulated AM signal waveform: transmitted AM signal at $0^{0}$

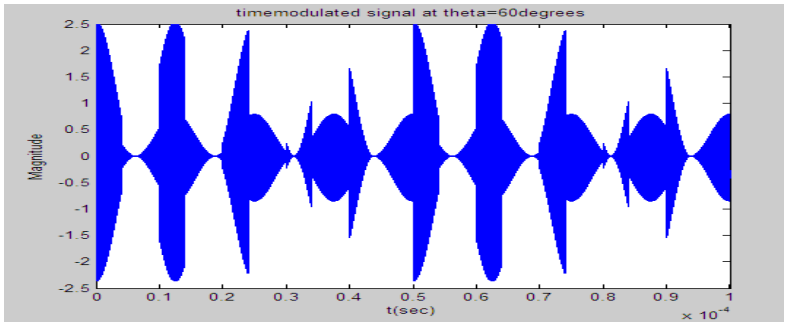

Fig.16. Simulated AM signal waveform: transmitted AM signal at $60^{\circ}$

In the numerical simulation, it is assumed that $\mathrm{f}_{\mathrm{s}}=80 \mathrm{KHz}$ and $\mathrm{f}_{0}=2.6 \mathrm{GHz}$. The time-modulation frequency is set as $\mathrm{f}_{\mathrm{p}}=100 \mathrm{KHz}$, which is less than the AM signal bandwidth $\left(B=2 f_{s}=160\right.$ KHz). Fig.9, Fig.10 \& Fig.11 illustrates the simulated frequency spectrum for the original signal and the transmitted signals at $0^{\circ}$ and $60^{\circ}$. It is observed in Fig. 9 $\&$ Fig. 10 that the shape of the simulated spectrum at broadside (i.e., $0^{0}$ ) is the same as that of the original AM signal, implying that the radiated signal at broadside is not time-modulated and correct. However, the spectrum for the signal at $60^{\circ}$ becomes overlapped due to time modulation, as shown in Fig.11. Even if a band-pass filter with a bandwidth $\mathrm{B}$ is used to filter out the unwanted sideband signals, the filtered spectrum shown in Fig.12 is still distorted as compared to that of the original signal. It should be noted that if $f_{p}<B$, the spectrum will be overlapped as long as there are sideband signals caused by time modulation.

To observe the transmitted signals directly, the timedomain waveforms of the transmitted signals are also simulated. Fig.13 illustrates the waveform of the original AM signal, Fig.14, Fig.15 \& Fig.16 illustrates the transmitted signals at $0^{\circ}, 25^{\circ}$, and $60^{\circ}$. As can be seen, the signal envelope shown in Fig.14 is almost the same as that in Fig.13. This means that the signal transmitted at $0^{0}$ (broadside) is correct. At the same time, the wave forms of transmitted signals at $25^{\circ}$ and $60^{\circ}$ have been timemodulated and become distorted due to the spectrum aliasing effects. Even if they are filtered, the waveforms still cannot be fully recovered. This is a consequence of the fact that the centre frequency and sideband signals are overlapped together and cannot be separated from each other.

\section{CONCLUSION}

The suitability of 4-D arrays as enabling technology for the antenna front-end of CR systems has been discussed. It has been shown that a proper definition of the pulse sequence controlling the on-off behavior of the RF switches enables the possibility to modulate the signal in order to transmit the correct information in a desired 
direction and make the signal un intelligible for eavesdroppers receiving the signal in other directions. As long as the transmitted signal bandwidth is much greater than the time modulation frequency, the signals transmitted through the side-lobes are distorted due to aliasing effect, while the signal at broadside is correct. Consequently, a secure communication can be achieved by using the 4-D arrays. An important part is to design a real proto type of a TMLA. A good attempt will be starting of designing an 8 or 16 elements linear array of dipole antennas with a simple feed network, and each array element could be switched on and off by the RF switches that are controlled by an external FPGA board.

\section{REFERENCES}

[1]. M. P. Daly and J. T. Bernhard, "Directional modulation technique for phased arrays," IEEE no. 9, pp. 2633-2640, Sep. 2009.

[2]. M. P. Daly, E. L. Daly, and J. T. Bernhard, "Demonstration of directional modulation using a phased array," IEEE Trans. Antennas Propag., vol. 58, no. 5, pp. 1545-1550, May 2010.

[3]. M.P.Daly and J.T.Bernhard, "Beam steering in pattern reconfigurable arrays using directional modulation," IEEE Trans. Antennas Propag., vol. 58, no. 7, pp. 2259-2265, Jul. 2010.

[4]. J.C.Brégains, J.Fondevila, G.Franceschetti, and F.Ares, "Signalradiation and power losses of time-modulated arrays," IEEE Trans. Antennas Propag., vol. 56, no. 6, pp. 1799-1804, Jun. 2008.

[5]. S.Yang, Y.B.Gan, and A.Qing, "Side band suppression in timemodulated linear arrays by the differential evolution algorithm," IEEE Antennas Wireless Propag. Lett.,vol.1, pp. 173-175, 2002.

[6]. J.Fondevila, J.C.Brégains, F.Ares, and E.Moreno, "Optimizing uniformly excited linear arrays through time modulation," IEEE Antennas Wireless Propag. Lett., vol. 3, pp. 298-301, 2004.

[7]. L.Poli, P. Rocca, L.Manica, and A.Massa, "Handling sideband radiations in time-modulated arrays through particle swarm optimization," IEEE Trans. Antennas Propag., vol. 58, no. 4, pp. 1408-1411, Apr. 2010.

[8]. G. Li, S. Yang, and Z. Nie, "Direction of arrival estimation in time modulated linear arrays with unidirectional phase center motion," IEEE Trans. Antennas Propag., vol. 58, no. 4, pp. 1105-1111, Apr. 2010.

[9]. L.Poli, P.Rocca, G.Oliveri, and A.Massa, "Harmonic beam forming in time-modulated linear arrays," IEEE Trans. Antennas Propag., vol. 59, no. 7, pp. 2538-2545, Jul. 2011.

[10]. T. Hong, M.-Z. Song, and Y. Liu, "RF directional modulation technique using a switched antenna array for physical layer secure communication applications," Progress in Electromagn. Res., vol.116, pp. 195-213, 2011 